\title{
Pulsating Solitons in Mode-Locked Fibre Lasers
}

\author{
Junsong Peng', Sonia Boscolo², Nikita Tarasov², Srikanth Sugavanam², Dmitry V. Churkin ${ }^{3}$, \\ Christophe Finot ${ }^{4}$ \\ ${ }^{I}$ State Key Laboratory of Precision Spectroscopy, East China Normal University, Shanghai, China \\ ${ }^{2}$ Aston Institute of Photonic Technologies, Aston University, Birmingham B4 7ET, United Kingdom \\ ${ }^{3}$ Novosibirsk State University, Novosibirsk 630090, Russia \\ ${ }^{4}$ Laboratoire Interdisciplinaire Carnot de Bourgogne, UMR 6303 CNRS - Université de Bourgogne Franche- \\ Comté, F-21078 Dijon Cedex, France \\ e-mail: s.a.boscolo@aston.ac.uk
}

\begin{abstract}
We report on our direct experimental observation of a new regime of operation of passively mode-locked fibre lasers where the laser oscillator generates pulsating solitons with extreme ratios of maximal to minimal intensities in each period of pulsations. The soliton spectra also experience large periodic broadening and compression. Spatio-temporal intensity and dispersive Fourier-transformation measurements enable us to capture such transient dynamics in real time.
\end{abstract}

Keywords: Dissipative nonlinear systems, mode-locked fibre lasers, extreme soliton pulsations.

\section{INTRODUCTION}

Mode-locked fibre lasers, besides being attractive sources of ultra-short optical pulses for many applications, constitute an ideal platform for the fundamental exploration of complex dissipative nonlinear dynamics. Various striking nonlinear phenomena have been experimentally observed in mode-locked fibre lasers, including rogue waves [1-4], soliton molecules [5], and soliton explosions [6]. Further, a recent theoretical work using the master-equation approach [7] has showed that there are regimes of operation where laser oscillators may generate pulsating solitons with extreme ratios of maximal to minimal energies in each period of pulsations. The soliton spectra in these regimes also experience large periodic variations. In this paper, we report on the direct experimental observation of extreme soliton pulsations in a passively mode-locked fibre laser. We capture these kinds of pulsations temporally and spectrally in real time using spatio-temporal intensity dynamics measurements and the dispersive Fourier transformation (DFT) $[8,9]$.

The methodology of spatio-temporal dynamics has recently enabled substantial progress in the real-time characterisation of dynamic, non-stationary generation regimes of lasers and soliton interactions in other cavitybased systems [10-12] owing to the possibility of identifying and tracking individual features embedded in the radiation that are otherwise hidden in the usual one-dimensional intensity measurements [13]. In this method, the intensity evolution in the laser is traced as it makes round-trip circulations within the laser cavity. Employing wide-bandwidth real-time digital storage oscilloscopes, it is possible to use long-term records of onedimensional intensity versus time information to experimentally arrive at a round-trip resolved, two-dimensional intensity domain representation of the laser dynamics. In the context of fibre lasers, such a technique has helped observe the laminar-turbulent transition in a laser [14], reveal non-trivial periodicity and long-scale correlations of radiation in partially mode-locked lasers [15], observe soliton instabilities in a passively mode-locked laser [6], and investigate different lasing regimes in quasi-continuous-wave Raman lasers [16]. DFT [8,9] is a relatively simple but powerful technique to obtain spectral dynamics rather than temporal information on ultrashort time scales: Spectral information is mapped into the time domain - using chromatic dispersion in a long optical fibre - and is detected via a high-speed photo-detector and real-time digitisation. This method is increasingly used for spectral measurements of rapid signals across a wide range of diverse application fields and, in recent experiments with mode-locked sources, has been applied to record the build-up of femtosecond mode locking [17] and the internal dynamics of soliton molecules [5,18]. The combination of the real-time spatio-temporal methodology with real-time DFT spectral measurements can provide a time-aligned spatio- and spectro-temporal characterisation of highly dynamic processes - a feat that remains possible only in numerical simulations.

\section{EXPERIMENTAL SETUP AND RESULTS}

The laser used in our experiment is a mode-locked erbium-doped fibre (EDF) ring laser, sketched in Fig. 1. The laser cavity incorporates a $1.5-\mathrm{m}$ long EDF with normal group-velocity dispersion $65.05 \mathrm{ps}^{2} / \mathrm{km}^{2} 1550 \mathrm{~nm}$, which is pumped through a 980/1550 wavelength-division multiplexer by a 976-nm laser diode. The other fibres are standard single-mode fibres (SMF-28) with a total length of $18.5 \mathrm{~m}$, yielding an anomalous path-averaged cavity dispersion of $-15.31 \mathrm{ps}^{2} / \mathrm{km}$. The mode-locking mechanism is nonlinear polarisation rotation, facilitated through the inclusion of a combination of two polarisation controllers (PCs) and an in-fibre polarisationdependent isolator. A 10:90 fibre coupler extracts 10\% of the in-cavity power. 
At the laser output, we record the spatio-temporal intensity evolution $I(t, T)$, i.e. the evolution of the instantaneous intensity pattern $I(t)$ over many cavity round-trips $T$, by a $80-\mathrm{GSa} / \mathrm{s}$ real-time oscilloscope (Agilent) together with a $50-\mathrm{GHz}$ fast photo-detector (Finisar). We also measure the ensemble-averaged spectrum using an optical spectrum analyser (OSA) and use the DFT to acquire high-resolution spectral measurements at the shot-to-shot level. The DFT is implemented by temporally stretching the laser output in a normally dispersive fibre of length $L \approx 11 \mathrm{~km}$, providing a total accumulated dispersion of $|D| L=1200 \mathrm{ps} / \mathrm{nm}$. This maps the pulse spectra into the temporal domain, allowing us to measure them in real time. Overall, our DFT configuration allows shot-to-shot measurements with a resolution of $\Delta \lambda=\Delta t /|D| L=0.025 \mathrm{~nm}$, where $\Delta t$ (30 ps) is the response time of the system.

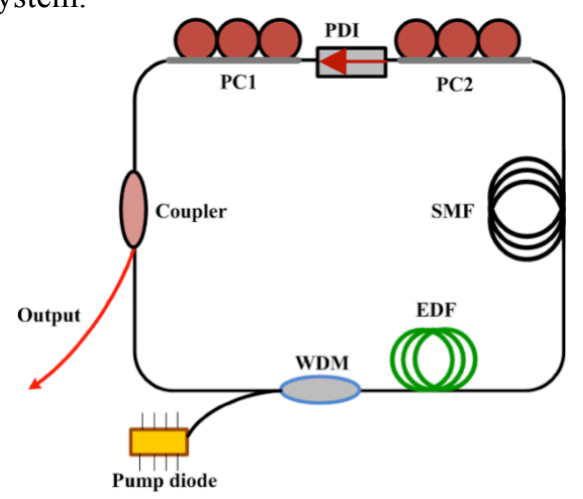

Figure 1. Schematic of the passively mode-locked fibre laser, WDM: wavelength-division multiplexer; EDF: erbium-doped fibre; SMF: single-mode fibre: PC: polarisation controller; PDI: polarisation-dependent isolator.

Depending on the rotation of the PCs and the pump level, this laser can sustain various emission regimes, including single-pulse soliton mode locking, soliton bunch generation, noise-like pulsing emission, and harmonic mode locking. In particular, we observe an unusual regime in which the laser oscillator generates multiple solitons whose intensity changes drastically and periodically as seen on the oscilloscope. In order to trace such dynamics, we performed spatio-temporal intensity measurements: we recorded a long-term trace of the laser output (thousands of consecutive pulse emissions) and we divided the recorded real-time signal into segments whose duration equals the average cavity round-trip time (approximately $600 \mathrm{ps}$ for this laser). We then concatenated all the segments into a single false colour plot. The results we have found for a quadruplebound soliton state are shown in Fig. 2(a), and we can clearly see that the four solitons experience large periodic variations in their intensity with a period of approximately 1100 cavity round trips. The peak intensity within each period of pulsations changes by nearly an order of magnitude, while the pulse width remains almost constant [Fig. 2(b)]. Further, the solitons exhibit a periodic shift of their temporal position. This is ascribed to a periodic change in the pulse repetition period following the temporal intensity variations (which affect the refractive index of the fibre). In this example, the temporal separation between closest neighbouring solitons is approximately $50 \mathrm{ps}$.

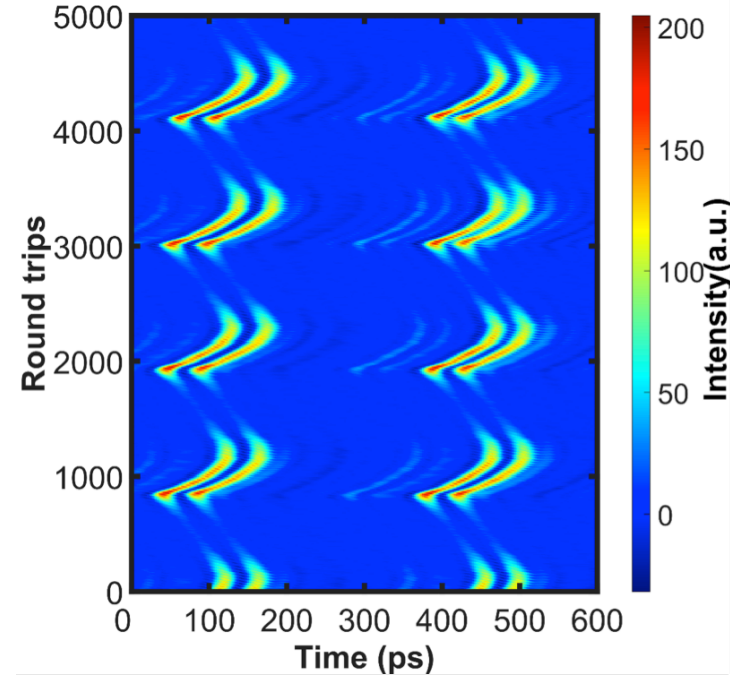

(a)

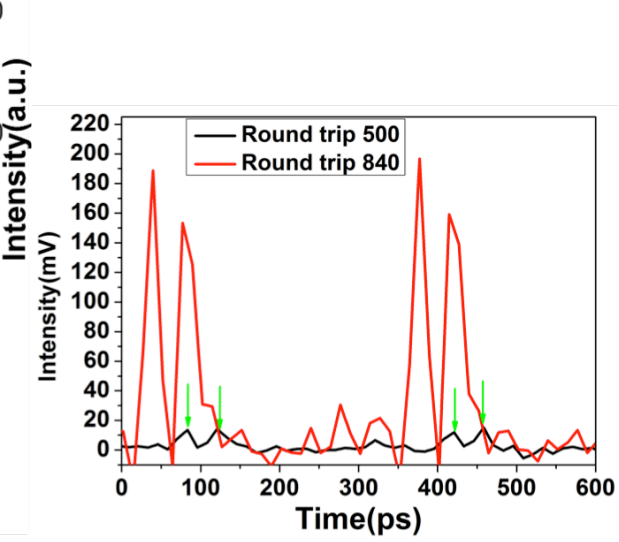

(b)

Figure 2. Experimentally measured temporal evolution of the intensity of a quadruple-soliton pulse relative to the average round-trip time over 5000 consecutive round trips. (b) Temporal intensity profiles at the round-trip numbers of maximal and minimal intensities within a period of oscillations. 
The corresponding average optical spectrum recorded with the OSA is shown in the top panel of Fig. 3(a), and is modulated as a result of the close pulse separation in time. We also used the DFT to measure the shot-to-shot spectra in real time. Figure 3(a) concatenates thousands of experimentally measured single-shot spectra of consecutive pulse emissions. It reveals that the evolution of the spectrum over cavity round trips is also periodic, and the spectrum largely widens and narrows within each period, with the broadening (compression) naturally occurring in the vicinity of the position where the solitons reach the highest (lowest) peak intensity. The maximum width of the spectrum exceeds the minimum width by several times [Fig. 3(b)]. The security of our implementation of the DFT technique is confirmed by the excellent agreement between the calculated averaged spectrum from the measured consecutive single-shot spectra and the spectrum recorded with the OSA.

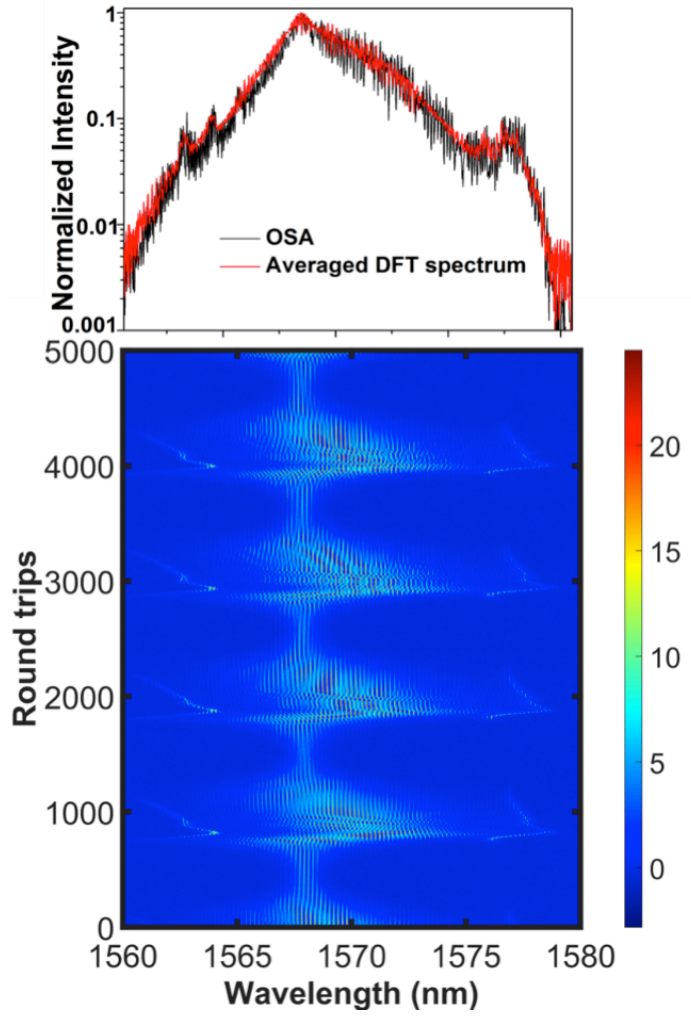

(a)

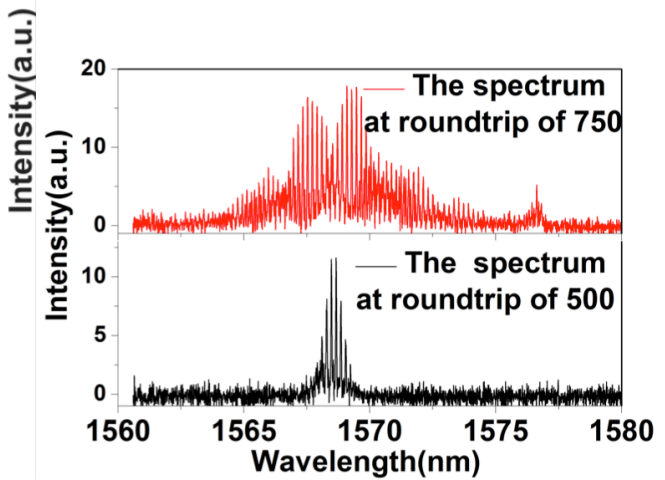

(b)

Figure 3. (a) Bottom: Experimentally measured single-shot spectra of 5000 consecutive quadruple-soliton pulses. Top: The calculated averaged spectrum from the single-shot spectra is compared with the average spectrum recorded with an OSA. (b) Spectra at the round-trip numbers of maximal and minimal spectrum extents within a period of oscillations.

Pulsations of triple- and double-soliton pulses are also observed in this laser cavity, although the pulsating behaviour is less pronounced than that of the quadruple-soliton case.

\section{CONCLUSIONS}

We have reported on the experimental observation of a strongly pulsating regime of multiple solitons in an anomalous-dispersion passively mode-locked fibre laser, through real-time spatio-temporal intensity dynamics measurements and DFT-based single-shot spectral measurements. In this regime, the solitons experience significant changes in their intensity and exhibit a concomitant time shift within a period of about 1000 cavity round trips $(\sim 0.1 \mathrm{~ms})$. Meanwhile their optical spectrum widens and narrows periodically. Our observations are, to the best of our knowledge, the first of their kind in a laser system. We anticipate that the occurrence of strong soliton pulsations is not unique to the specific fibre laser cavity discussed above. Indeed, we have experimentally observed strongly pulsating solitons in other cavity realisations. Numerical simulations of the laser dynamics based on a realistic piece-wise model for the laser cavity are currently performed to confirm the experiments and provide additional insight and incentive for future investigations. Our findings suggest that extremely pulsating solitons can pave the way for the realisation of new ultrafast light sources of high peak power.

\section{ACKNOWLEDGEMENTS}

European Commission Marie Curie International Incoming Fellowship (628198), ERC project ULTRALASER, H2020 project CARDIALLY, and the Russian Foundation for Basic Research (16-32-60153). 


\section{REFERENCES}

[1] C. Lecaplain, et al.: Dissipative rogue waves generated by chaotic pulse bunching in a mode-locked laser, Phys. Rev. Lett., vol. 108, pp. 233901, 2012.

[2] A.F.J. Runge, et al.: Raman rogue waves in a partially mode-locked fiber laser, Opt. Lett., vol. 39, pp. 319322, 2014.

[3] Z. Liu, S. Zhang, F.W. Wise: Rogue waves in a normal-dispersion fiber laser, Opt. Lett., vo. 40, pp. 13661369, 2015.

[4] M. Liu, et al.: Dissipative rogue waves induced by long-range chaotic multi-pulse interactions in a fiber laser with a topological insulator-deposited microfiber photonic device, Opt. Lett., vol. 40, pp. 4767-4770, 2015.

[5] K. Krupa, et al:: Real-time observation of dissipative optical soliton molecular motions, arXiv preprint arXiv:1702.01161, 2017.

[6] A.F.J. Runge, N.G.R. Broderick, M. Erkintalo: Observation of soliton explosions in a passively modelocked fiber laser, Optica, vol. 2, pp. 36-39, 2015.

[7] W. Chang, et al.: Extreme soliton pulsations in dissipative systems, Phys. Rev. E, vol. 92, 022926, 2015.

[8] D.R. Solli, J. Chou, B. Jalali: Amplified wavelength-time transformation for real-time spectroscopy, Nat. Photon., vol. 2, pp. 48-51, 2008.

[9] K. Goda, B. Jalali: Dispersive Fourier transformation for fast continuous single-shot measurements, Nat. Photon., vol. 7, pp. 102-112, 2013.

[10] M. Marconi, et al.: Vectorial dissipative solitons in vertical-cavity surface-emitting lasers with delays, Nat. Photon., vol. 9, pp. 450-455, 2015.

[11] J. K. Jang, et al.: Ultraweak long-range interactions of solitons observed over astronomical distances, Nat. Photon., vol. 7, pp. 657-663, 2013.

[12] M. Erkintalo, et al:: Bunching of temporal cavity solitons via forward Brillouin scattering, New J. Phys., vol. 17, 115009, 2015.

[13] F.T. Arecchi, et al.: Two-dimensional representation of a delayed dynamical system, Phys. Rev. A, vol. 45, R4225(R), 1992.

[14] E. G. Turitsyna, et al.: The laminar-turbulent transition in a fibre laser, Nat. Photon., vol. 7, pp. 783-786, 2013.

[15] D.V. Churkin, et al., Stochasticity, periodicity and localized light structures in partially mode-locked fibre lasers, Nat. Commun., vol. 6, 7004, 2015.

[16] N. Tarasov, S. Sugavanam, D. Churkin: Spatio-temporal generation regimes in quasi-CW Raman fiber lasers, Opt. Express, vol. 23, pp. 24189-24194, 2015.

[17] G. Herink, et al.: Resolving the build-up of femtosecond mode-locking with single-shot spectroscopy at 90 MHz frame rate, Nat. Photon., vol. 10, pp. 321-326, 2016.

[18] G. Herink, et al.: Real-time spectral interferometry probes the internal dynamics of femtosecond soliton molecules, Science, vol. 356, pp. 50-54, 2017. 